Check for updates

Cite this: Mater. Chem. Front., $2019,3,284$

Received 28th November 2018, Accepted 11th December 2018

DOI: $10.1039 / c 8 q m 00613 j$

rsc.li/frontiers-materials

\section{A novel strategy for realizing dual state fluorescence and low-temperature phosphorescence $\uparrow$}

\author{
Yunxiang Lei, ${ }^{a}$ Wenbo Dai, ${ }^{a}$ Zhiqi Liu, ${ }^{a}$ Shuai Guo, ${ }^{a}$ Zhengxu Cai, (D) *a \\ Jianbing Shi, (DD ${ }^{a}$ Xiaoyan Zheng, ${ }^{* b}$ Junge Zhi, (DD ${ }^{b}$ Bin Tong ${ }^{a}$ and Yuping Dong ${ }^{* a}$
}

\begin{abstract}
We demonstrate a new strategy for the development of dual-state emissive materials by introducing phenyl groups to triphenylpyrrole (TPP) isomers. The phenyl group can increase the rotational barrier and restrict the intramolecular rotation of TPP, thus enhancing the fluorescence efficiency of aggregation induced emission (AIE) materials in solution. Fluorescence of TPP derivatives was not quenched by the drastic intermolecular collision and intramolecular motion at high temperature. On the contrary, the fluorescence of their solutions showed a positive response to temperature. Theoretical calculations demonstrated that the molecules exhibited a higher planar conformation at high temperature. Three donor-acceptor samples with a twisted intramolecular charge transfer (TICT) effect showed a strong phosphorescence with a lifetime up to $10 \mathrm{~s}$ at low temperature.
\end{abstract}

\section{Introduction}

Organic conjugated fluorogens are widely applied in chem/ biosensors, bioimaging, and optoelectronics because of their excellent performances, such as facile preparation, easy modification, high fluorescence brightness, and tunable spectral properties. $^{1-6}$ Most organic fluorogens only exhibit strong luminescence in solution (aggregation-causing quenching, ACQ) or in the solid state form (aggregation-induced emission, AIE), which has prevented further realization of their potential applications. $^{7-13}$ Recently, researchers developed fluorogens with strong fluorescence in both solid and liquid states, namely, dual-state emission (DSE) materials. ${ }^{14-18}$ Exploration of DSE materials can overcome the issues associated with ACQ and AIE materials and broaden overall applications of fluorogens in both solution and solid states. ${ }^{14,15}$ However, how to realize the DSE property and improve their luminescence efficiency is an intriguing question that has no straightforward answers. A rigid conjugation backbone can increase the intrinsic luminescence of organic conjugated molecules. However, this large conjugation resulting in a condensed molecular packing can quench the luminescence in the solid state. ${ }^{7,8}$ Combinatorial approaches to the discovery of DSE materials have emerged as exciting alternatives for the exploration and optimization of

\footnotetext{
${ }^{a}$ School of Materials Science \& Engineering, Beijing Institute of Technology, Beijing, 100081,China.E-mail: caizx@bit.edu.cn,chdongyp@bit.edu.cn ${ }^{b}$ School of Chemistry and Chemical Engineering, Beijing Institute of Technology, Beijing, 100081, China. E-mail: xiaoyanzheng@bit.edu.cn

$\dagger$ Electronic supplementary information (ESI) available. CCDC 1853745-1853750. For ESI and crystallographic data in CIF or other electronic format see DOI: 10.1039/c8qm00613j
}

fluorogens. A delicate balance between twisting conformation and rigid conjugation endows organic conjugated molecules with a strong fluorescence intensity in both solution and the solid state. $^{16-18}$ In addition, high temperature can weaken or even quench the fluorescence due to the increase of intramolecular motion and intermolecular collisions, which is another factor to lower the luminescence efficiency. Thus, it is of great practical significance to study fluorescent materials with positive responses to elevated temperatures. ${ }^{19-24}$ Several strategies were adopted to avoid the fluorescence quenching at high temperatures. Wang et al. reported a series of $\mathrm{Pt}^{\mathrm{II}}$-based frameworks with a positive response to temperature because of the intermolecular excimer formation. ${ }^{19}$ Thermally activated delayed fluorescent (TADF) compounds also showed a higher fluorescence intensity at high temperatures because excitons lose their spin-forbidden states at these temperatures, leading to intersystem crossing (ISC). However, a low ISC efficiency results in low quantum yields of TADF materials. $^{22-24}$ Despite considerable efforts to develop novel organic fluorogens, DSE materials with a high luminescence efficiency are still underexplored due to their unclear luminescence mechanism. Therefore, in-depth theoretical research and guidance are still needed.

Recently, our group developed a series of polyarylpyrroles such as triphenylpyrrole isomers (TPP-1,2,4, TPP-1,2,5 and TPP$1,3,4)$, tetraphenylpyrrole (TePP) and pentaphenylpyrrol (PPP) (Scheme 1). ${ }^{25-27}$ Their AIE and ACQ as well as DSE properties were determined by the position of the corresponding phenyl substitution. ${ }^{27}$ A phenyl group attached to the 1-position of the pyrrole ring can act as a rotor that consumes the energy of the excited state. Thus, removing a phenyl group attached to the $\mathrm{N}$ atom would effectively enhance the molecular emission in solution 


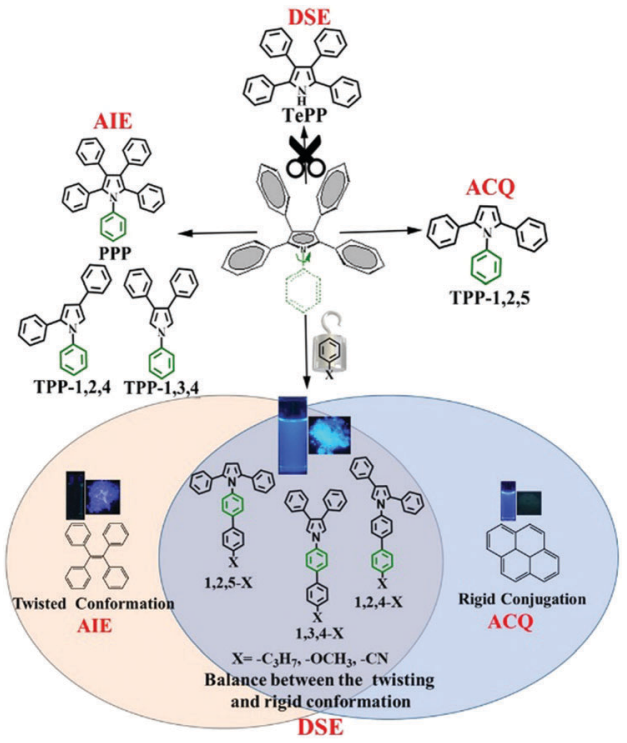

Scheme 1 The strategy for realizing dual state emission properties.

allowing TePP to have DSE properties. ${ }^{27}$ On the other hand, as the volume of the group at the 1-position increases, the corresponding rotational barriers also increase, which will effectively restrict the intramolecular motion, allowing materials to have bright fluorescence in solution. Based on this strategy, we designed and synthesized nine compounds demonstrating $33-55 \%$ absolute quantum yields in both solution and solid states. The optical properties of these compounds are dependent on the ambient temperature. Fluorescence intensity is significantly enhanced as the temperature increases. In addition, three donor (D)-acceptor (A) compounds show strong phosphorescence in both solution and solid states at low temperatures with a lifetime of up to $10 \mathrm{~s}$.

\section{Results and discussion}

\section{Synthesis and characteristics of TPP-based compounds}

Scheme 2 illustrates molecular structures of the nine triphenylpyrrole (TPP) derivatives. All TPP-based molecules showed absorption bands in the $240-310 \mathrm{~nm}$ range and emission bands in the $365-515 \mathrm{~nm}$ range (Fig. 1 and Fig. S1, ESI $\dagger$ ). The highest occupied molecular orbital (HOMO) and the lowest unoccupied molecular orbital (LUMO) are shown in Fig. S5 (ESI $\dagger$ ) and their energy levels are summarized in Table 1. The HOMO and LUMO of three TPP-based derivatives with $\mathrm{CN}$ groups are spatially separated, indicating the existence of intermolecular charge transfer (ICT) (Fig. S2, ESI†).

\section{DSE properties of TPP-based compounds}

Our previous results demonstrated that the phenyl group at the 1-position of the pyrrole ring acts as a rotor, consumes the excited state energy and lowers the emission in solution. ${ }^{27}$ Therefore, the increase in the rotational barrier at the 1-position will effectively restrict the intramolecular motion allowing the materials to have bright fluorescence in solution (Scheme 1). This strategy can change the TPP-based molecules with both AIE and
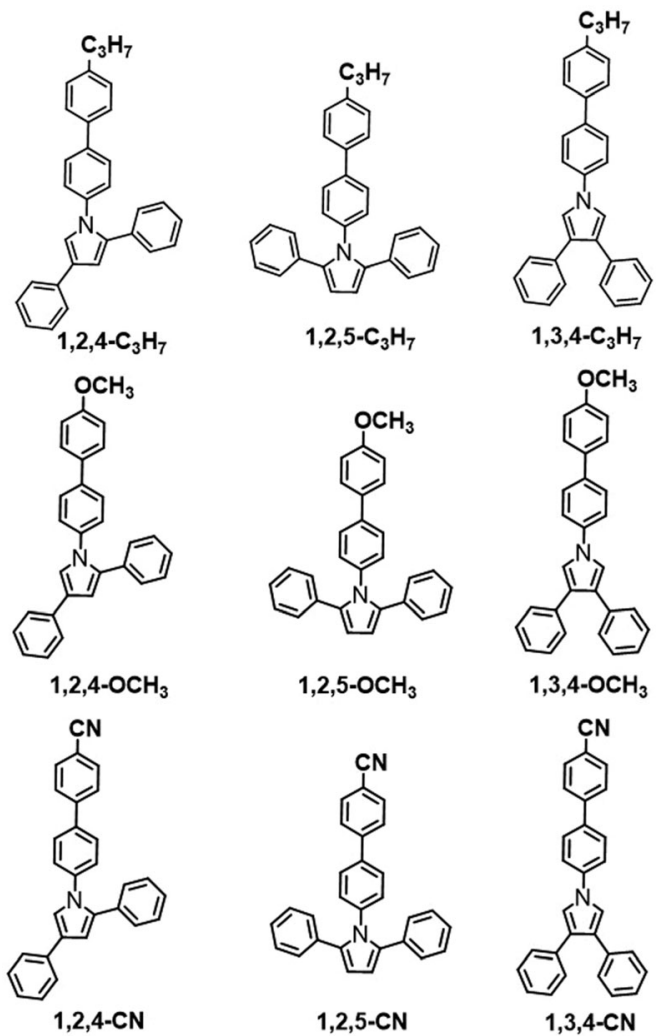

Scheme 2 Molecular structures of organic conjugated fluorogens.
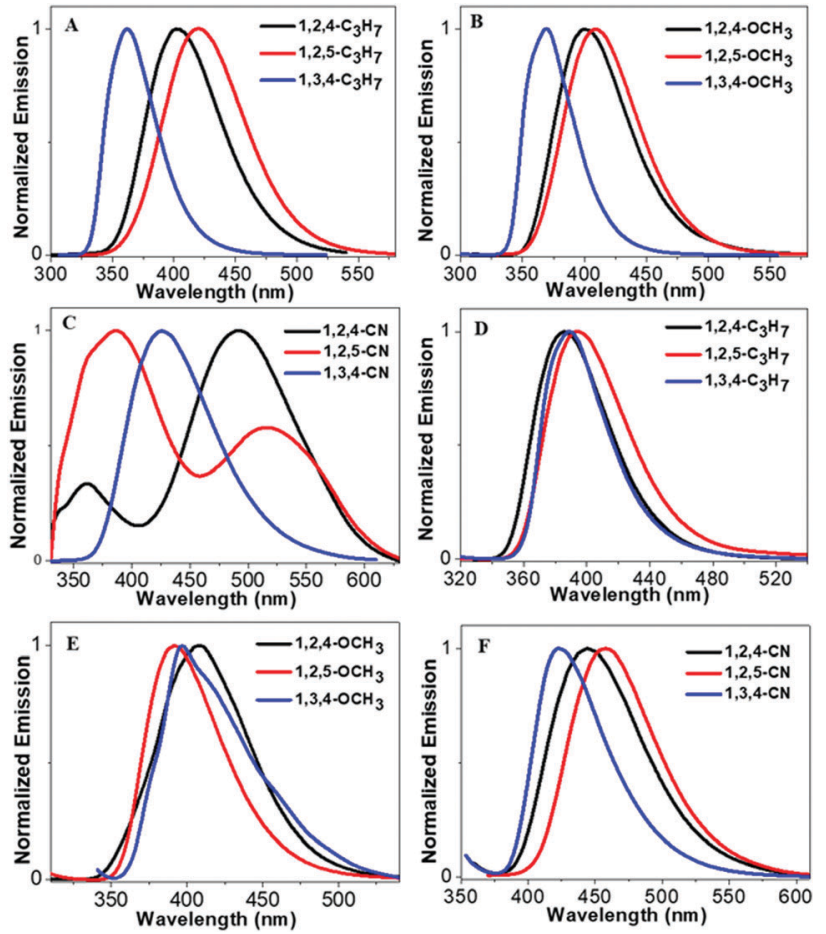

Fig. 1 (A-C) Emission spectra of TPP-based derivatives in THF solution (concentrations: $10^{-5} \mathrm{M}$ ), and $(D-F)$ emission spectra of TPP-based derivatives in the solid state. 
Table 1 Photophysical properties of the TPP-based molecules

\begin{tabular}{|c|c|c|c|c|c|c|c|c|c|c|c|c|}
\hline Samples & $\begin{array}{l}\lambda_{\mathrm{em}}(\mathrm{nm}) \\
(\mathrm{THF})\end{array}$ & $\begin{array}{l}\lambda_{\mathrm{em}}(\mathrm{nm}) \\
\text { (solid) }\end{array}$ & $\begin{array}{l}\Phi_{\mathrm{F}}(\%) \\
(\mathrm{THF})\end{array}$ & $\begin{array}{l}\Phi_{\mathrm{F}}(\%) \\
\text { (solid) }\end{array}$ & $\begin{array}{l}E_{\mathrm{g}} \\
(\mathrm{eV})\end{array}$ & $\begin{array}{l}\text { Dipole } \\
\text { moment/DB }\end{array}$ & $\begin{array}{l}\tau(\mathrm{ns}) \\
(\mathrm{THF})\end{array}$ & $\begin{array}{l}\tau(\mathrm{ns}) \\
\text { (solid) }\end{array}$ & $\begin{array}{l}k_{\mathrm{r}}\left[10^{-8} \mathrm{~s}^{-1}\right] \\
(\mathrm{THF})\end{array}$ & $\begin{array}{l}k_{\mathrm{nr}}\left[10^{-8} \mathrm{~s}^{-1}\right] \\
(\mathrm{THF})\end{array}$ & $\begin{array}{l}k_{\mathrm{r}}\left[10^{-8} \mathrm{~s}^{-1}\right] \\
\text { (solid) }\end{array}$ & $\begin{array}{l}k_{\mathrm{nr}}\left[10^{-8} \mathrm{~s}^{-1}\right] \\
\text { (solid) }\end{array}$ \\
\hline $1,2,4-\mathrm{C}_{3} \mathrm{H}_{7}$ & 402 & 389 & 43.2 & 48.2 & 4.25 & 3.09 & 3.06 & 1.71 & 1.41 & 1.86 & 2.82 & 3.03 \\
\hline $1,2,5-\mathrm{C}_{3} \mathrm{H}_{7}$ & 418 & 393 & 32.0 & 42.1 & 4.11 & 2.74 & 3.36 & 2.57 & 0.95 & 2.02 & 1.64 & 2.25 \\
\hline $1,3,4-\mathrm{C}_{3} \mathrm{H}_{7}$ & 365 & 391 & 44.3 & 44.5 & 4.34 & 3.16 & 2.09 & 1.18 & 2.12 & 2.67 & 3.77 & 4.70 \\
\hline $1,2,4-\mathrm{OCH}_{3}$ & 404 & 399 & 47.1 & 45.7 & 4.21 & 3.48 & 2.32 & 1.17 & 2.03 & 2.28 & 3.91 & 4.64 \\
\hline $1,2,5-\mathrm{OCH}_{3}$ & 410 & 395 & 36.6 & 40.1 & 4.16 & 3.23 & 2.68 & 1.79 & 1.37 & 2.37 & 2.24 & 3.35 \\
\hline $1,3,4-\mathrm{OCH}_{3}$ & 367 & 393 & 43.6 & 47.1 & 4.36 & 3.72 & 1.81 & 0.96 & 2.41 & 3.12 & 4.91 & 5.51 \\
\hline $1,2,4-\mathrm{CN}$ & $360 / 492$ & 444 & 47.6 & 53.5 & 3.03 & 3.83 & 5.04 & 6.87 & 0.94 & 1.04 & 0.78 & 0.68 \\
\hline $1,2,5-\mathrm{CN}$ & $386 / 515$ & 458 & 32.8 & 49.6 & 2.95 & 3.62 & 6.72 & 7.23 & 0.49 & 1.00 & 0.69 & 0.70 \\
\hline $1,3,4-\mathrm{CN}$ & 425 & 423 & 55.6 & 44.3 & 3.26 & 3.90 & 4.32 & 5.04 & 1.29 & 1.03 & 0.88 & 1.11 \\
\hline
\end{tabular}

ACQ properties to molecules with DSE properties by simply extending the conjugation length at the 1-position of the pyrrole molecule. All molecules showed a high fluorescence QY (33-55\%) both in liquid and solid states indicating their DSE properties (Table 1 and Fig. 2). To further study the luminescence properties of compounds in the aggregated state, emission behaviors of their $\mathrm{THF} / \mathrm{H}_{2} \mathrm{O}$ mixtures were investigated.

Fluorescence intensities of the aggregates of all TPP derivatives (measured in $\mathrm{THF} / 99 \% \mathrm{H}_{2} \mathrm{O}$ ) were almost identical to those in THF solution, indicating their DSE properties (Fig. S3-S5, ESI†). The maximum emission wavelengths of all molecules redshift as there is an increase in the water fraction, which is likely due to the high polarity of water. However, the maximum emission wavelengths of all molecules except $1,3,4-\mathrm{C}_{3} \mathrm{H}_{7}$ and $1,3,4-\mathrm{OCH}_{3}$ were then blue-shifted, when the aggregates were formed in higher water fractions. In addition, all compounds showed shorter emission wavelengths in solid states than those in solution except $1,3,4-\mathrm{C}_{3} \mathrm{H}_{7}$ and $1,3,4-\mathrm{OCH}_{3}$. This could be attributed to the through-space conjugation effect. This effect decreases energy reorganization and suppresses non-radiative decay processes in aggregated states, thereby causing strong and blue-shifted aggregated emissions. ${ }^{28-30}$ The phenyl rings at the 2,5-positions in 1,2,5-TPP based molecules overcome steric hindrance to form a favorable face-to-face geometry, leading to the longest blueshifts in the solid state. The blue-shifts of three 1,2,4-TPP based compounds in the solid state are smaller than those of 1,2,5-TPP

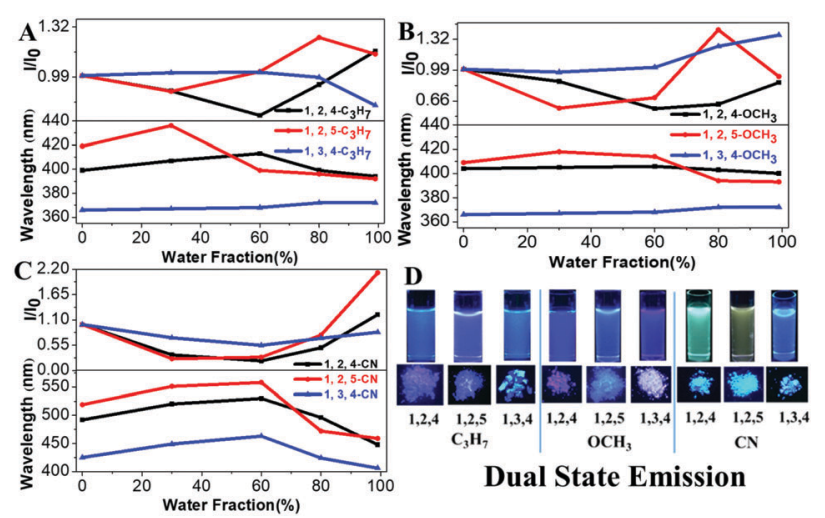

Fig. 2 (A-C) Fluorescence intensity of TPP derivatives in $\mathrm{THF} / \mathrm{H}_{2} \mathrm{O}$ mixtures with different water fractions. (D) Visible fluorescence of compounds under $302 \mathrm{~nm}$ UV light (concentrations: $10^{-5} \mathrm{M}$ ). based molecules because only one face-to-face geometry was formed. 1,3,4- $\mathrm{C}_{3} \mathrm{H}_{7}$ and 1,3,4- $\mathrm{OCH}_{3}$ showed red-shifts in the solid state due to the lack of effective through-space conjugation and intramolecular D-A interactions (Fig. S6, ESI†)).

\section{Single crystal structures}

Six of the nine compounds can be crystallized (Tables S3-S8, ESI $\dagger$ ), while $1,2,4-\mathrm{C}_{3} \mathrm{H}_{7}, 1,2,5-\mathrm{C}_{3} \mathrm{H}_{7}$ and $1,2,5-\mathrm{OCH}_{3}$ did not yield crystals that could be analyzed by XRD. The $1,2,4-\mathrm{OCH}_{3}$ molecule has a relatively distorted spatial configuration with poor planarity. Crystals of 1,2,4- $\mathrm{OCH}_{3}$ adopted a head-to-head cross-parallel packing pattern along the $b$-axis. The closest interaction distance of $\pi-\pi$ was $5.901 \AA$ and weak $\mathrm{C}-\mathrm{H} \cdots \pi$ interactions with a $2.977 \AA$ distance were observed (Fig. 3A-C). Crystals of 1,3,4-OCH 3 also adopted a head-to-head cross-parallel packing pattern along the $b$-axis. The closest $\pi-\pi$ and $\mathrm{C}-\mathrm{H} \cdots \pi$ interaction distances were 6.148 and $2.895 \AA$ in the solid state, respectively (Fig. 3E). Compound 1,3,4- $\mathrm{C}_{3} \mathrm{H}_{7}$ showed configuration and intermolecular arrangements similar to those for 1,3,4-OCH $\mathrm{OCH}_{3}$ (Fig. 3G-I), suggesting that the two compounds exhibit similar emission wavelengths and fluorescence intensities in the solid state (Table 1). TPP compounds with the $-\mathrm{CN}$ group showed different molecular configuration and intermolecular arrangements. The 1,2,4-CN molecule showed a distorted spatial configuration, and its crystal adopted a head-totail cross-vertical arrangement pattern along the $b$-axis. An obvious $\mathrm{C}-\mathrm{H} \cdots \pi$ interaction was observed with a distance of $2.891 \AA$ and $2.931 \AA$ A (Fig. 4A-C). 1,2,5-CN adopted a head-to-head arrangement pattern along the $b$-axis. The closest interaction distance of the $\pi-\pi$ plane was $6.058 \AA$, and a very weak $\mathrm{C}-\mathrm{H} \cdots \pi$ interaction in the crystal was observed with a distance of $3.273 \AA$ (Fig. 4D-F). A single crystal structure of 1,3,4-CN adopted a tail-to-tail cross-vertical arrangement pattern along the $b$-axis. The large twist angles of the 3,4-positioned benzene rings and the pyrrole core make the overall volume of the molecule relatively large. The distance between the closest faceto-face benzene rings reaches $4.002 \AA$ (Fig. 4G-I). Thus, all molecules show no close $\pi-\pi$ interactions, resulting in high luminescence efficiency of all the compounds in the solid state. In addition, twisted excited state conformations prevent the corresponding molecules from close packing which results in detrimental formations (such as excimers) and are helpful for aggregate emission. Theoretical calculations showed that the twist angles between the TPP moiety and the biphenyl plane are close to $90^{\circ}$ in excited states (Fig. S7, ESI $\dagger$ ). Perpendicular conformation of the entire molecule can effectively help to avoid 


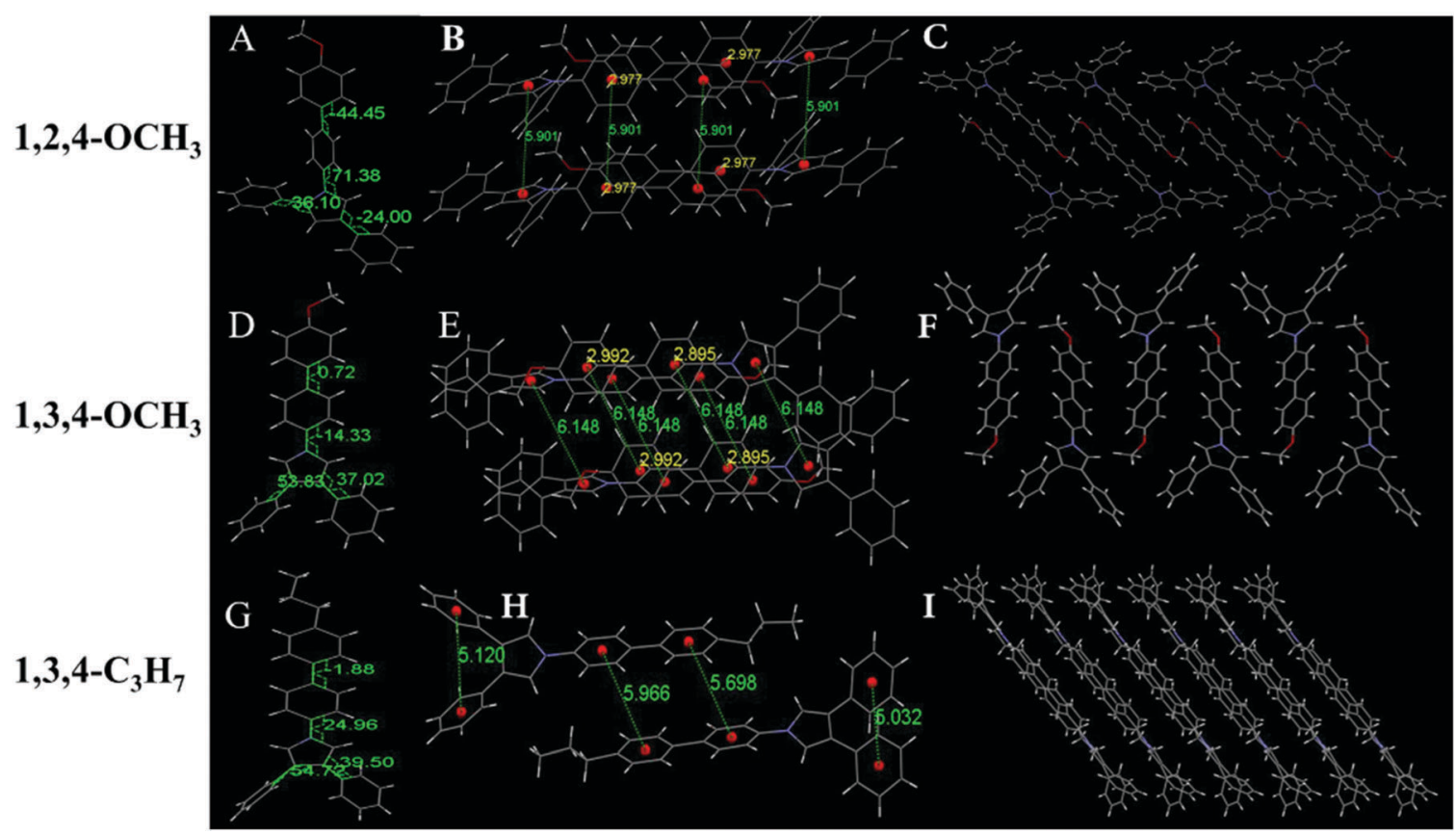

Fig. 3 (A) Molecule structure, (B) interaction distance of the $\pi \cdots \pi$ and $\mathrm{C}-\mathrm{H} \cdots \pi$ interactions and (C) stacking image along the $b$-axis of 1,2,4-OCH . (D) Molecular structure, (E) interaction distance of the $\pi \cdots \pi$ and $\mathrm{C}-\mathrm{H} \cdots \pi$ interactions and (F) stacking image along the $b$-axis of $1,3,4-\mathrm{OCH}$. (G) Molecular structure, $(\mathrm{H})$ interaction distance of the $\pi \cdots \pi$ and $\mathrm{C}-\mathrm{H} \cdots \pi$ interactions and (I) stacking image along the $b$-axis of $1,3,4-\mathrm{C}_{3} \mathrm{H}_{7}$. Gray color corresponds to carbon, white to hydrogen, red to oxygen and blue to nitrogen.

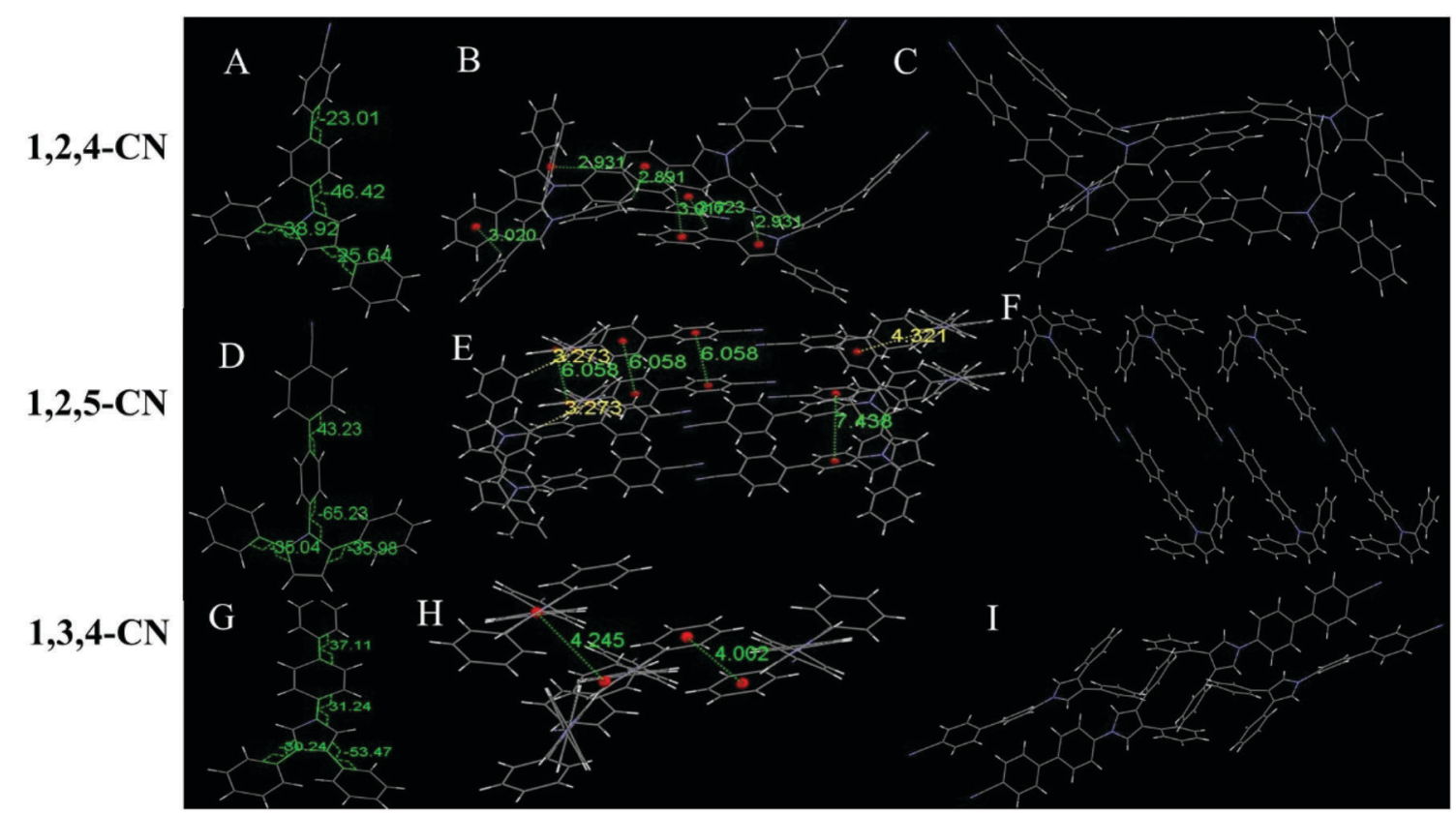

Fig. 4 (A) Molecular structure, (B) interaction distances of $\mathrm{C}-\mathrm{H} \cdots \pi$ interactions and (C) stacking image of 1,2,4-CN. (D) Molecular structure, (E) interaction distances of $\mathrm{C}-\mathrm{H} \cdots \pi$ interactions and (F) stacking image of 1,2,5-CN. (G) Molecular structure, (H) interaction distances of face-to-face phenyl rings and (I) stacking image of 1,3,4-CN. Gray color corresponds to carbon, white to hydrogen and blue to nitrogen.

the formation of the excitatory associations, allowing compounds to have strong fluorescence in the aggregated state. ${ }^{31,32}$

\section{Positive response to temperature}

Fluorescence of organic fluorogens is typically quenched at high temperature due to the increase of intramolecular motion and intermolecular collisions. Yet, our TPP-based molecules showed a positive response as temperature increased. Fig. 5A shows the fluorescence intensity of 1,3,4-CN at different temperatures $\left(10^{-5} \mathrm{M}\right.$ in THF). Fluorescence intensity increased as the temperature increased and decreased when the ambient temperature was lowered (Fig. S8, ESI $\dagger$ ). Similar phenomena 

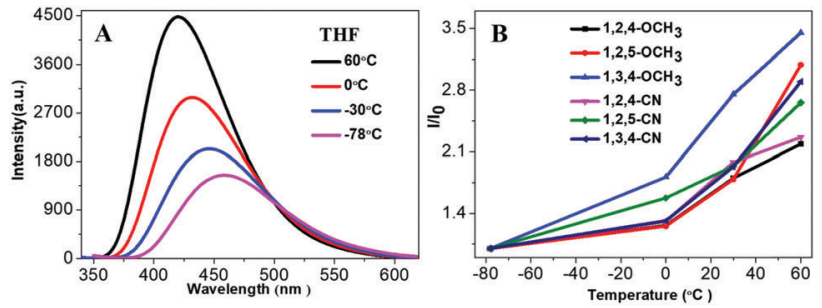

Fig. 5 (A) PL spectra of 1,3,4-CN solution and (B) relationship between the $\mathrm{PL}$ intensities of fluorogens $\left(10^{-5} \mathrm{M}\right.$ in THF) at different temperatures.

were also observed for $1,3,4-\mathrm{CN}$ in toluene or DMF solution (Fig. S9, ESI $\dagger$ ). However, the fluorescence intensity variations of $1,3,4-\mathrm{CN}$ in toluene or DMF solution were smaller than those in THF solution, indicating that the fluorescence enhancement was influenced by the solvent. All other compounds also demonstrated similar responses to the ambient temperature (Fig. 5B and Fig. $\mathrm{S} 10$, ESI $\dagger$ ). However, TPP-based derivatives with $\mathrm{C}_{3} \mathrm{H}_{7} / \mathrm{OCH}_{3}$ groups only showed a variation in fluorescence intensity due to the lack of intramolecular D-A interactions. Emission wavelengths of TPP-based derivatives with $\mathrm{CN}$ groups blue-shifted as the temperature increased because polarity of the solvent decreased as temperature increased (Fig. S11, ESI $\dagger$ ). ${ }^{33}$ Since the more polar excited state resulting from a charge transfer is more stable than the less polar ground state in a polar solvent, emission spectra shifted to shorter wavelengths as the temperature increased. ${ }^{33-38}$

To demonstrate the structure-absorption spectrum relationship for the 1,3,4-CN system, we scanned the profiles of wavelength and oscillator strength as a function of selected key dihedral angles (Fig. 6A and B). All scanned profiles were
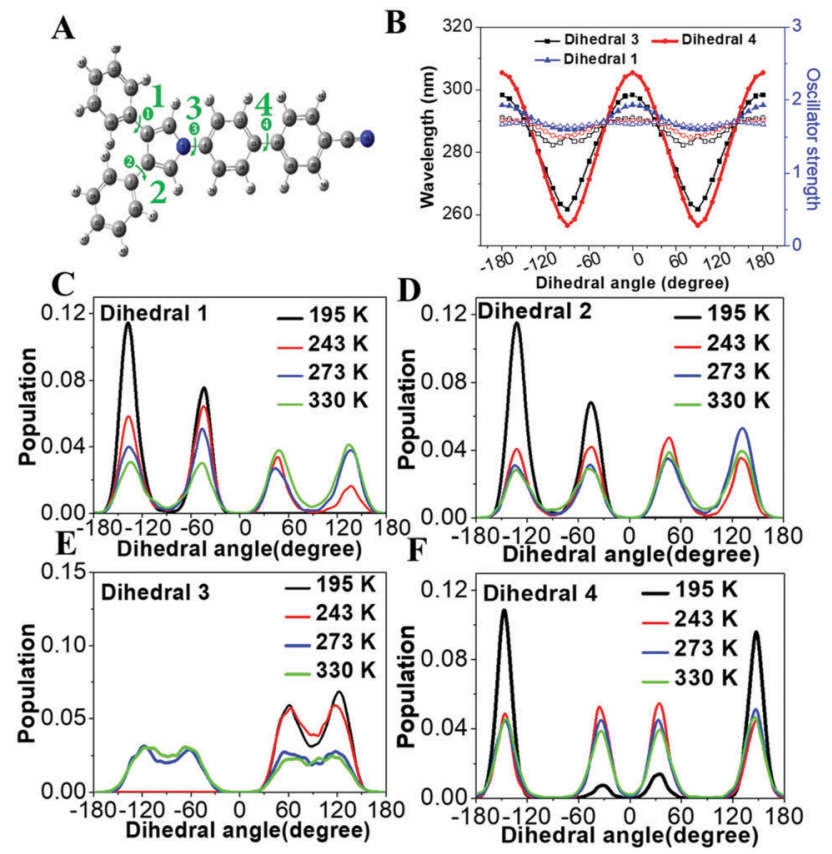

Fig. 6 (A) Optimized geometry of $1,3,4-\mathrm{CN}$ in the ground state $\left(\mathrm{S}_{0}, \mathrm{mim}\right)$ (B) Wavelength and oscillator strength profiles of $1,3,4-\mathrm{CN}$ as functions of the selected dihedral angles. (C-F) Distributions of selected dihedral angles in the 1,3,4-CN system in THF solution at 195, 243, 263 and $330 \mathrm{~K}$. prepared based on the optimized geometry of $1,3,4-\mathrm{CN}$ in the ground state at the CAM-B3LYP/6-31G(d) level. Except for the selected dihedral angles, all other degrees of freedom were frozen during scanning. Because of the symmetric property of dihedral angles 1 and 2, we only calculated profiles for dihedral angles 1, 3 and 4 (Fig. 6B). We found that: (1) the wavelength profiles of both dihedral angles 3 and 4 fluctuate in much larger amplitude than that of dihedral angle 1, indicating that dihedral angles 3 and 4 have a major impact on the absorption wavelength of $1,3,4-\mathrm{CN}$. This is also supported by the negligible overlap between the HOMO and LUMO of 1,3,4-CN on the TPP moiety (Fig. S3, ESI $\dagger$ ). (2) The oscillator strength, which reflects intensity of the absorption spectrum, fluctuates in a small range between 1.40 and 1.75. (3) For all dihedral angles, the better conjugation rotors show a longer absorption wavelength and the perpendicular rotors give the shortest wavelengths. For example, the wavelengths of dihedral angle 4 were 305 and $256 \mathrm{~nm}$ when it equals $0^{\circ}$ and $90^{\circ}$, respectively. Therefore, the absorption spectrum of $1,3,4-\mathrm{CN}$ should be determined by the statistical possibility of different conformations.

The molecular conformations change in different environments. To get more insights into the relationship between the conformations and absorption spectra of 1,3,4-CN, we performed molecular dynamics (MD) simulations to obtain molecular conformations of 1,3,4-CN at four different temperatures and under two solvent conditions. A single 1,3,4-CN molecule was placed in the center of a cubic box with pre-equilibrated THF or toluene molecules, respectively. ${ }^{39}$ For each solvent, we setup MD simulations at temperatures: 195, 243, 273 and $330 \mathrm{~K}$, respectively to analyze the temperature effect for the $1,3,4-\mathrm{CN}$ molecule. For each of the eight systems, we first performed energy minimization followed by 500 ps MD simulations under the NVT ensemble with position restraints on 1,3,4-CN. Then we performed two independent $50 \mathrm{~ns}$ MD simulations under the $N P T$ ensemble at $P=1$ bar and at the target temperature for each system. The temperature and pressure were controlled by the velocity rescaling thermostat and the Parrinello-Rahman barostat. The electrostatic interactions were evaluated using the particle mesh Ewald method. The cutoff distances for both direct space summation and the van de Waals interactions were $1.2 \mathrm{~nm}$. All bond lengths were constrained by the LINCS algorithm. ${ }^{40}$ Periodic boundary conditions were applied in all three dimensions to minimize the edge effects in a finite system. The time step was $2 \mathrm{fs}$. The conformations were stored at $40 \mathrm{ps}$ time intervals and in total $2500 \mathrm{MD}$ conformations were collected for each system to get dihedral angle populations. Obtained dihedral angle distributions of 1,3,4-CN in THF and toluene solution are plotted in Fig. 6C-F and Fig. S13 (ESI $\dagger$ ), respectively. From Fig. 6C-F, we found that: (1) the dihedral angle distribution is localized in a narrow range at lower temperature $(\sim 195 \mathrm{~K})$. At the same time, at $330 \mathrm{~K}$, dihedral angles are distributed in the whole range: from $-180^{\circ}$ to $180^{\circ}$. Dihedral angles 1 and 2 play a minor role in the change of absorption spectra (Fig. 6B) and similar distributions at different temperatures are observed. (2) As temperature increased from 195 to $330 \mathrm{~K}$, distributions of dihedral angle 4 between $-30^{\circ}$ and $30^{\circ}$ increased significantly. This is the key factor leading to the 

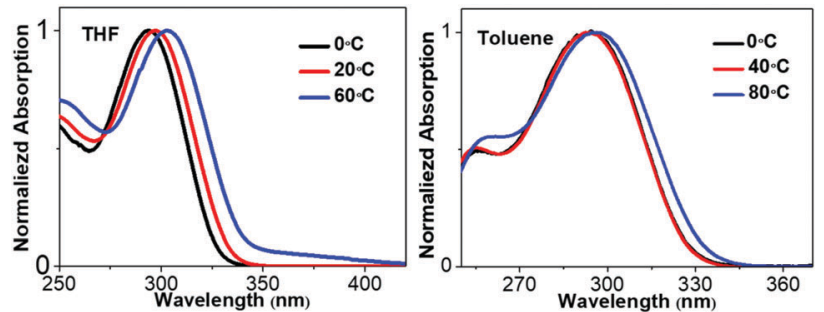

Fig. 7 Absorption spectra of 1,3,4-CN in the THF and toluene solution at different temperatures $\left(10^{-5} \mathrm{M}\right)$.

obvious red-shift of the absorption spectrum in THF solution (Fig. 7). (3) Distributions of dihedral angles 1, 2 and 3 of 1,3,4-CN in toluene solution were still local in a narrow range even at $343 \mathrm{~K}$ reflecting its less sensitive conformational change in toluene than that in THF. Therefore, the absorption spectrum of $1,3,4$-CN in toluene was less red-shifted than that in THF solution (Fig. 7). Thus, as temperature increases, the molecular conformation tends to planarize resulting in enhanced fluorescence of the compounds. The twisted conformation of 1,3,4-CN at low temperature will decrease the bandgap and the energy gap between the lowest singlet and the triplet states $\left(\Delta E_{\mathrm{ST}}\right)$ and increase the phosphorescence, as discussed below.

\section{Long lifetime phosphorescence property}

Three TPP derivatives with CN groups showed a more intense fluorescence emission and a shorter emission wavelength at high temperatures. However, they exhibited a pronounced phosphorescence emission at low temperatures both in solution and the solid state (Fig. 8 and 9). The phosphorescence wavelength of 1,2,5-CN in 2-methyl tetrahydrofuran solution was slightly longer than that of $1,2,4-\mathrm{CN}$. The phosphorescence wavelength for 1,3,4-CN was the shortest (Fig. 9). Three samples in 2-methyl tetrahydrofuran solution showed a phosphorescence lifetime as long as $10 \mathrm{~s}$ as well as high excitation a phosphorescence efficiency up to $11.75 \%$ (Fig. $8 \mathrm{~B}$ ) because of

A

\begin{tabular}{|l|l|c|c|c|c|c|c|c|}
\hline & \multicolumn{10}{c}{ UV OFF } \\
\hline
\end{tabular}

Fig. 8 (A) Luminescence photos of 1,2,4/1,2,5/1,3,4-CN and 1,3,4- $\mathrm{OCH}_{3}$ in 2-methyltetrahydrofuran solutions $\left(10^{-5} \mathrm{M}\right)$ at $77 \mathrm{~K}$ before and after UV irradiation at $365 \mathrm{~nm}$. (B) Optical properties of the TPP-based molecules.
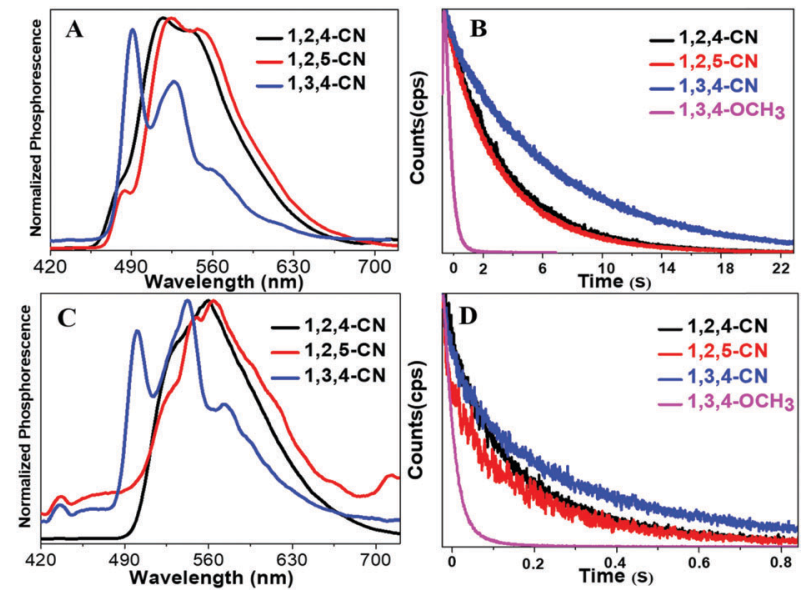

Fig. 9 Normalized phosphorescence spectra of 1,2,4/1,2,5/1,3,4-CN compounds in solution (A) and in the solid state (C). Time-resolved phosphorescence decay curves of $1,2,4 / 1,2,5 / 1,3,4-\mathrm{CN}$ and $1,3,4-\mathrm{OCH}_{3}$ in 2-methyltetrahydrofuran solutions $\left(10^{-5} \mathrm{M}\right)$ at $77 \mathrm{~K}(\mathrm{~B})$ in the solid state (D).

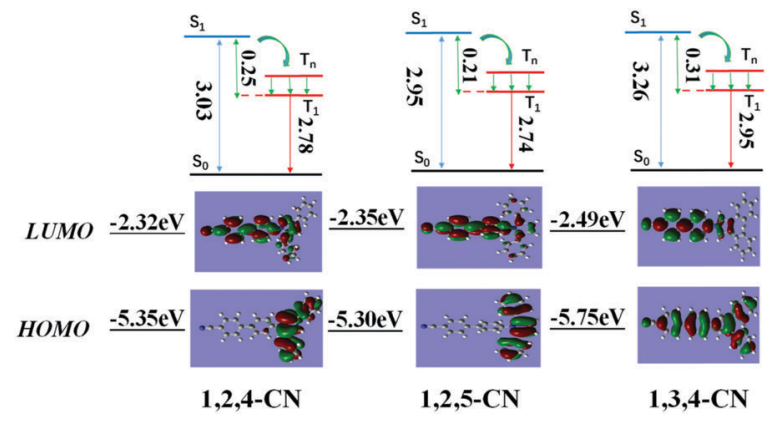

Fig. 10 Calculated energy diagrams and HOMO/LUMO orbital distributions of TPP based molecules with the $\mathrm{CN}$ group.

the small energy gap $\left(\Delta E_{\mathrm{ST}}\right.$ : the energy gap between the lowest singlet and triplet states). Typically, small values of $\Delta E_{\mathrm{ST}}$ are beneficial for the improvement of the intersystem energy crossing. ${ }^{41-43}$ Spatial separation of the HOMO and LUMO can lead to small $\Delta E_{\mathrm{ST}}$ in twisted D-A systems. ${ }^{44-46}$ Therefore, three D-A molecules with ICT effects have excellent low-temperature phosphorescence properties. Phosphorescence wavelengths of three compounds are consistent with their energy gaps between the lowest triplet and the ground states $\left(\Delta E_{\mathrm{TG}}\right)$ (Fig. $8 \mathrm{~B}$ and 10 ). The other six molecules without ICT effects showed higher $\Delta E_{\mathrm{ST}}$ values (Table S2, ESI $\dagger$ ), indicating that it is difficult for them to achieve intersystem crossing, thus showing no phosphorescence emission. In addition, three samples also showed phosphorescence in the solid state at $T=77 \mathrm{~K}$ with a short life time and a low QY, probably due to the intermolecular interactions of the compounds in the solid state. This causes the energy of the triplet state to return to the ground state in a non-radiative manner. ${ }^{45-49}$

\section{Conclusions}

In this work, we synthesized a series of TPP-based molecules, all of which showed DSE properties with quantum yields over $30 \%$. 
As the temperature increased, the molecules became flatter, which increased their fluorescence intensity. MD simulations showed that the fluorescence increase was caused by the variation of molecular conformation, indicating rigid molecular conformations of DSE molecules. Three TPP molecules with CN groups showed a low-temperature phosphorescence up to $10 \mathrm{~s}$ demonstrating a great opportunity for the development of long life-time materials.

\section{Conflicts of interest}

There are no conflicts to declare.

\section{Acknowledgements}

This work was financially supported by the National Natural Scientific Foundation of China (Grant No. 51803009, 51673024, 51328302 and 21404010), the Graduate Technological Innovation Project of Beijing Institute of Technology (2018CX10005), the Beijing Institute of Technology Research Fund Program for Young Scholars and the Graduate Technological Innovation Project of Beijing Institute of Technology.

\section{Notes and references}

1 H. N. Kim, Z. Guo, W. Zhu, J. Yoon and H. Tian, Chem. Soc. Rev., 2011, 40, 79-93.

2 Z. X. Cai, N. Zhang, M. A. Awais, A. S. Filatov and L. P. Yu, Angew. Chem., Int. Ed., 2018, 57, 6442-6448.

3 H. Nie, K. Hu, Y. Cai, Q. Peng, Z. Zhao, R. Hu, J. Chen, S. J. Su, A. Qin and B. Z. Tang, Mater. Chem. Front., 2017, 1, 1125-1129.

4 J. Mei, Y. Hong, J. W. Y. Lam, A. J. Qin, Y. H. Tang and B. Z. Tang, Adv. Mater., 2014, 26, 5429-5479.

5 Z. X. Cai, W. Y. Lo, T. Y. Zheng, L. W. Li, N. Zhang, Y. B. Hu and L. P. Yu, J. Am. Chem. Soc., 2016, 138, 10630-10635.

6 Y. X. Lei, H. Li, M. C. Liu, J. X. Chen, X. B. Huang and H. Y. Wu, J. Mater. Chem. C, 2014, 2, 7402-7410.

7 S. A. Jenekhe and J. A. Osaheni, Science, 1994, 265, 765-768. 8 H. Shi, H. Sun, H. Yang, S. Liu, G. Jenkins, W. Feng, F. Li, Q. Zhao, B. Liu and W. Huang, Adv. Funct. Mater., 2013, 23, 3268-3276.

9 Y. X. Lei, Y. Y. Lai, L. C. Dong, J. B. Shi, J. G. Zhi, X. B. Huang and Y. P. Dong, Chem. - Eur. J., 2018, 24, 434-442.

10 G. Chen, W. B. Li, T. R. Zhou, Q. Peng, D. Zhai, W. Z. Yuan, Y. M. Zhang and B. Z. Tang, Adv. Mater., 2015, 27, 4496-4501.

11 F. D. Nisi, R. Francischello, A. Battisti, A. Panniello, E. Fanizza, B. Tang and A. Pucci, Mater. Chem. Front., 2017, 1, 1406-1412.

12 B. Chen, Z. J. Zhao and B. Z. Tang, Chin. Sci. Bull., 2016, 61, 3435-3447.

13 Z. Peng, Z. Wang, B. J. Shi, B. Tong and Y. P. Dong, Chin. J. Chem., 2016, 34, 1071-1075.

14 T. Beppu, K. Tomiguchi, A. Masuhara, Y. J. Pu and H. Katagiri, Angew. Chem., Int. Ed., 2015, 54, 7332-7335.

15 M. N. Huang, R. N. Yu, S. X. Ye, S. Kang, X. H. Zhu and Y. Q. Wan, Chem. Sci., 2016, 7, 4485-4491.
16 K. Suenaga, K. Tanaka and Y. Chujo, Eur. J. Org. Chem., 2017, 5191-5196.

17 M. Li, Y. L. Niu, X. Z. Zhu, Q. Peng, H. Y. Lu, A. D. Xia and C. F. Chen, Chem. Commun., 2014, 50, 2993-2995.

18 Y. Y. Li, Y. X. Lei, L. C. Dong, J. G. Zhi, J. B. Shi, B. Tong and Y. P. Dong, Chem. - Eur. J., DOI: 10.1002/chem.201804074.

19 L. J. Liu, X. Wang, N. Wang, T. Peng and S. Wang, Angew. Chem., Int. Ed., 2017, 59, 9160-9164.

20 P. O. Sersson, T. Sergeim, R. L. Chen and T. Gillbro, J. Phys. Chem., 1995, 99, 16199-16209.

21 Q. Y. Fang, J. W. Li, S. Y. Li, S. Q. Wang, Y. P. Yi, Y. Qian and G. Q. Yang, Chem. Commun., 2017, 53, 5702-5705.

22 Z. Y. Yang, Z. Mao, Z. L. Xie, Y. Zhang, J. Zhao, Z. G. Chi and M. P. Aldred, Chem. Soc. Rev., 2017, 46, 915-1016.

23 T. Hatakeyama, K. Shiren, S. Nomura, S. Nakatsuka, Y. Ono and T. Ikuta, Adv. Mater., 2016, 28, 2777-2781.

24 A. J. Qin, Y. Zhang, N. Han, J. Mei, S. J. Zhi and B. Z. Tang, Sci. China: Chem., 2012, 55, 772-778.

25 L. C. Dong, G. J. Shang, J. B. Shi, J. G. Zhi, B. Tong and Y. P. Dong, J. Phys. Chem. C, 2017, 121, 11658-11664.

26 X. Feng, B. Tong, J. B. Shi, L. Chen, J. G. Zhi, P. Lu, Y. G. Ma and Y. P. Dong, J. Phys. Chem. B, 2010, 114, 16731-16736.

27 Y. X. Lei, Q. W. Liu, L. C. Dong, J. B. Shi, J. G. Zhi, Z. X. Cai and Y. P. Dong, Chem. - Eur. J., 2018, 24, 14269-14274.

28 H. Zhang, X. Y. Zheng, N. Xie, Z. K. He, Y. K. Sing, T. K. Kwok, H. H. Y. Sung, I. D. Williams, A. J. Qin and B. Z. Tang, J. Am. Chem. Soc., 2017, 139, 16264-16272.

29 S. J. Zhen, J. C. Mao, L. Chen, S. Y. Ding, X. S. Zhou, A. J. Qin, Z. J. Zhao and B. Z. Tang, Nano Lett., 2018, 18, 4200-4205.

30 W. W. Luo, H. Nie, B. R. He, Z. J. Zhao, Q. Peng and B. Z. Tang, Chem. - Eur. J., 2017, 23, 18041-18048.

31 Z. Q. Xie, B. Yang, F. Li, G. Cheng, H. Xu, M. Hanif, S. Y. Liu, D. G. Ma and Y. G. Ma, J. Am. Chem. Soc., 2005, 127, 14152-14153.

32 M. Gon, K. Tanaka and Y. Chujo, Angew. Chem., Int. Ed., 2018, 57, 6546-6551.

33 K. Li, Y. Y. Liu, Y. Y. Li, Q. Feng, H. W. Hou and B. Z. Tang, Chem. Sci., 2017, 8, 7258-7267.

34 H. Li, Y. Guo, Y. X. Lei, W. X. Gao, M. C. Liu, J. X. Chen, Y. F. Hu, X. B. Huang and H. Y. Wu, Dyes Pigm., 2015, 112, 105-115.

35 Y. H. Niu, Q. Wang, H. R. Wu, Y. X. Wang and Y. R. Zhang, New J. Chem., 2017, 41, 9796-9805.

36 X. D. Jiang, X. Liu, T. Fang and C. L. Sun, Dyes Pigm., 2017, 146, 438-444.

37 J. Do, J. Huh and E. Kim, Langmuir, 2009, 25, 9405-9412.

38 H. J. Liu, X. J. Xu, Z. J. Shi, K. Q. Liu and Y. Fang, Anal. Chem., 2016, 88, 10167-10175.

39 G. Bussi, D. Donadio and M. Parrinello, J. Chem. Phys., 2007, 126, 014101.

40 B. Hess, H. Bekker, H. J. C. Berendsen and J. E. M. Fraaije, J. Comput. Chem., 1997, 18, 1463-1472.

41 Y. Xiong, Z. Zhao, W. J. Zhao, H. L. Ma, Q. Peng, Z. K. He, X. P. Zhang, J. W. Y. Lam and B. Z. Tang, Angew. Chem., Int. Ed., 2018, 57, 7997-8001.

42 O. Bolton, K. Lee, H. J. Kim, K. Y. Lin and J. Kim, Nat. Chem., 2011, 3, 205-210. 
43 Z. F. An, C. Zheng, Y. Tao, R. F. Chen, H. F. Shi, T. Chen, Z. X. Wang, H. H. Li, R. R. Deng and W. Huang, Nat. Mater., 2015, 14, 685-689.

44 L. Gu, H. F. Shi, M. G. Gu, K. Ling, H. L. Ma, S. Z. Cai, L. L. Song, Z. G. Shuai and W. Huang, Angew. Chem., Int. Ed., 2018, 57, 8425-8431.

45 Y. Y. Hu, Z. F. Wang, X. F. Jiang, X. Y. Cai, S. J. Su, F. Huang and Y. Cao, Chem. Commun., 2018, 54, 7850-7853.
46 Y. Gong, L. Zhao, Q. Peng, D. Fan, W. Z. Yuan, Y. Zhang and B. Z. Tang, Chem. Sci., 2015, 6, 4438-4444.

47 Z. Z. Zhang, L. L. Tang, X. J. Fan, Y. H. Wang, S. F. Xue and W. J. Yang, J. Mater. Chem. C, 2018, 6, 8984-8989.

48 Y. Takeda, T. Kaihara, M. Okazaki, H. Higginbotham, P. Data, N. Tohnai and S. Minakata, Chem. Commun., 2018, 54, 6847-6850. 49 S. F. Pan, Z. T. Chen, X. L. Zheng, D. H. Wu, H. Feng and Z. S. Qian, J. Phys. Chem. Lett., 2018, 9, 3939-3945. 